\title{
Single/dual-wavelength switchable bidirectional Q- switched all-fiber laser using a bidirectional fiber polarizer
}

\author{
CHUANHANG ZOU ${ }^{1}$, QIANQIAN HUANG ${ }^{1}$, TIANXING WANG ${ }^{1,2}$, ZHIJUN \\ YAN $^{3}$, MOHAMMED AL ARAIMI4,5,6, ALEKSEY ROZHIN ${ }^{4,5}$, AND CHENGBO \\ MOU ${ }^{1, *}$
}

\author{
${ }^{1}$ Key Laboratory of Specialty Fiber Optics and Optical Access Networks, Shanghai Institute for Advanced Communication and Data Science, Joint \\ International Research Laboratory of Specialty Fiber Optics and Advanced Communication, Shanghai University, Shanghai 200444, P. R. China \\ ${ }^{2}$ Key Laboratory of Materials for High Power Laser, Shanghai Institute of Optics and Fine Mechanics, Chinese Academy of Sciences, Shanghai 201800, \\ P.R. China \\ ${ }^{3}$ School of Optical and Electronic Information, National Engineering Laboratory for Next Generation Internet Access System, Huazhong University of \\ Science and Technologies, Wuhan 430074, P.R. China \\ ${ }^{4}$ Aston Institute of Photonic Technologies (AIPT), Aston University, Birmingham, B4 7ET, United Kingdom \\ ${ }^{5}$ Nanoscience Research Group, Aston University, Birmingham, B4 7ET, United Kingdom \\ ${ }^{6}$ Al Musanna College of Technology, Muladdah, Al Musanna, P.O.Box 191, P.C.314, Sultanate of Oman \\ *Corresponding author: mouc1@shu.edu.cn
}

Received XX Month XXXX; revised XX Month, XXXX; accepted XX Month XXXX; posted XX Month XXXX (Doc. ID XXXXX); published XX Month XXXX

A single/dual-wavelength switchable bidirectional Qswitched fiber laser using a bidirectional fiber polarizer is demonstrated. $45^{\circ}$ tilted fiber grating $\left(45^{\circ} \mathrm{TFG}\right)$ is used as a bidirectional fiber polarizer to induce bidirectional intracavity birefringence filter in both clockwise (CW) and counter-clockwise (CCW) directions. Carbon nanotube saturable absorber (CNT-SA) is employed to produce Q-switched pulses. Through adjusting polarization states, switchable single/dual wavelength lasing at $1551 \mathrm{~nm}$, and $1560 \mathrm{~nm}$ can be achieved in both CW and CCW directions. To the best of our knowledge, this is the first demonstration of wavelength switchable bidirectional passively Q-switched fiber laser. (C) 2018 Optical Society of America

OCIS codes: (060.3735) Fiber Bragg gratings; (140.3510) Lasers, fibers; (140.3540) Lasers, Q-switched; (160.4236) Nanomaterials.

http://dx.doi.org/10.1364/OL.99.099999

Bidirectional pulsed fiber lasers with the abilities to generate pulses simultaneously in both clockwise (CW) and counterclockwise (CCW) directions can find important applications in the fields of gyroscopes, sensing [1] and dual-comb spectroscopy [2]. Since Khanh Kieu et al demonstrated the first all-fiber bidirectional mode-locked laser based on carbon nanotubes [1], various types of all-fiber bidirectional mode-locked lasers have been demonstrated [3-6]. Due to the discrepancy in nonlinearity, gain and loss caused by the asymmetry of a bidirectional operated laser cavity, pulses generated in CW and CCW direction may feature distinct properties from each other in terms of frequency, pulse duration, output power and spectra. Nevertheless, a dualwavelength bidirectional mode-locked fiber laser was demonstrated in Ref [7], which can operate at different wavelength window in CW and CCW direction as a result of different gain profile between counter-propagating directions. On the other hand, bidirectional Q-switched fiber lasers also have attracted attentions, because they may provide neat solutions in areas such as sum-frequency mixing and synthesis of optical waveform [5, 8, 9]. Moreover, multi-wavelength Q-switched fiber lasers can have wide applications including airborne Lidar, terahertz generation, optical communication and microwave radiation $[10,11]$. It is scientifically and technically interesting to implement control over lasing wavelength from a single laser cavity, especially when the lasing directionality will have been considered. Multi-wavelength bidirectional Q-switched fiber laser is firstly observed in Ref [5], however, control on lasing wavelength had not been explained in detail. Also, the Q-switching operation in ref [5] was an intermediate state from such laser. As a consequence of different gain profile, mode competition and asymmetry of a bidirectional operated ring laser cavity, it can be difficult to flexibly achieve more than one lasing wavelength from a stable bidirectional Q-switched fiber laser [5, 7]. 
In standard unidirectional operated laser cavities, due to mode competition resulted from homogeneous gain broadening of the erbium-doped fiber (EDF) at room temperature [12], laser cavities are less prone to produce multiple wavelengths. Some methods based on alleviating mode competition in homogeneous gain broadening are demonstrated to generate dual-wavelength such as techniques based on polarization hole burning (PHB) effect [13] and four-wave mixing (FWM) effect [14]. Alternatively, when incorporating a nonlinear saturable absorber device, wavelength selective devices can be used to realize multi-wavelength operation of the fiber laser including fiber Bragg grating [11], fiber taper [15]. Compared with the filters mentioned above, fiber based birefringence filters [16] provide a better way to realize multiwavelength generation with flexibly controlled performance.

Usually, a polarizer such as polarization dependent isolator combining with intrinsic fiber birefringence is able to form a fiber birefringence filter in unidirectional ring fiber laser [17]. However, it is somehow difficult to obtain two obvious fiber birefringence filters in both CW and CCW directions of bidirectional ring fiber lasers. Bidirectional polarizer such as polarizing beam splitters (PBS), single polarization fiber (SPS) [18]can solve this problem. But PBS will destroy the all-fiber structure of the laser and SPS would induce extra loss. Fortunately, $45^{\circ}$ tilted fiber grating ( $45^{\circ} \mathrm{TFG}$ ) is able to couple the $s$ light out of the fiber core and let the $p$ light pass through the fiber core with negligible loss, thus $45^{\circ} \mathrm{TFG}$ can be employed as a kind of ideal fiber polarizer with the advantages of low insertion loss, high polarization dependent loss (PDL) [19]. Moreover, light can be linearly polarized from any end of the $45^{\circ} \mathrm{TFG}$. Therefore, $45^{\circ} \mathrm{TFG}$ can be used as an effective bidirectional fiber polarizer, which can be employed in bidirectional fiber laser to induce bidirectional fiber birefringence filter.

In this letter, we demonstrate a single/dual-wavelength switchable bidirectional Q-switched all-fiber laser based on a $45^{\circ} \mathrm{TFG}$ and carbon nanotube saturable absorber (CNT-SA). CNTSA plays the role of generating Q-switched pulses and $45^{\circ} \mathrm{TFG}$ functions as a bidirectional polarizer that is used to help induce the bidirectional intracavity birefringence filter. Through adjusting the two intracavity polarization controllers (PCs), the bidirectional Qswitched fiber laser can operate at switchable single /dualwavelength of $1551 \mathrm{~nm}$ and $1560 \mathrm{~nm}$ in both CW and CCW direction. To the best of our knowledge, this is the first demonstration of a wavelength switchable bidirectional Qswitched fiber laser. It may have potential applications in the fields of dual-laser source design, synthesis of optical waveform, terahertz generation etc.

Fig. 1. exhibits the schematic configuration of the demonstrated bidirectional Q-switched fiber laser setup. The fiber laser consists of $0.83 \mathrm{~m}$ EDF (OFS, EDF 80) as gain fiber, $0.48 \mathrm{~m}$ wavelength division multiplexer (WDM) with pigtailed fiber (OFS 980) and $3.49 \mathrm{~m}$ standard single mode fiber (SMF), the total length of the laser cavity is $4.8 \mathrm{~m}$. The EDF is pumped by a $980 \mathrm{~nm}$ diode laser (OVLINK) through WDM. A small piece of carbon nanotube/ polyvinyl alcohol (CNT-PVA) composite film with 31\% nonsaturable loss and $6 \%$ modulation depth is incorporated between two standard fiber ferrules to act as an all-fiber saturable absorber. Detailed fabrication method of the CNT-PVA composite film can be found in elsewhere[20]. The used CNT is single walled and commercial available, which is fabricated by high pressure carbon monoxide conversion. A home-made $45^{\circ} \mathrm{TFG}$ combined with intracavity fiber birefringence can realize birefringence comb filter. The PDL of the $45^{\circ} \mathrm{TFG}$ at $1550 \mathrm{~nm}$ is $16 \mathrm{~dB}$ which is high enough to act as an in-fiber bidirectional polarizer [19]. The absence of optical isolator and a $40 \% 2 \times 2$ output coupler (OC) therefore help realize bidirectional operation of the fiber laser.

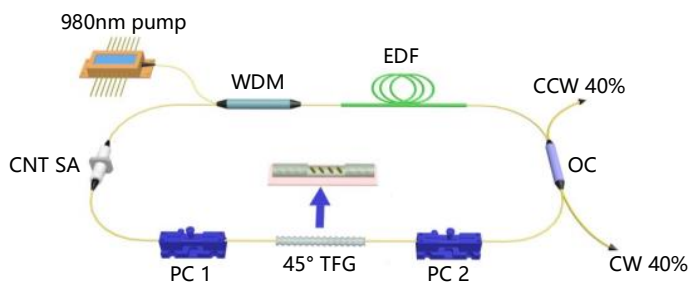

Fig. 1. Experimental configuration of the bidirectional fiber laser.
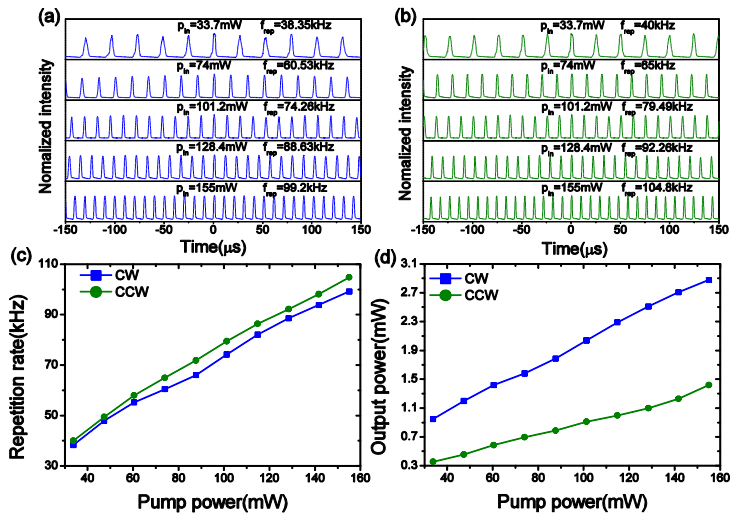

Fig. 2. Pulse trains evolution under different pump power: (a) in CW direction, and (b) in CCW direction; (c) Pulse repetition rate and (d) output power evolution versus pump power in CW and CCW directions.

When the pump power increases to $13.3 \mathrm{~mW}$, Q-switched pulses can be generated simultaneously in both CW and CCW direction at $1560 \mathrm{~nm}$. Fig. 2. (a) and (b) shows the pulses train evolution under different pump power in both CW and CCW directions, respectively. It can be clearly observed that with the elevation of pump power, the number of pulses increases correspondingly in both lasing directions. The evolution of repetition rate and output power versus pump power is depicted in Fig. 2. (c) and (d), respectively. As shown in Fig. 2. (c) and (d), when the pump power increases from $33.7 \mathrm{~mW}$ to $155 \mathrm{~mW}$, the repetition rate monotonically increases from $38.35 \mathrm{kHz}$ to 99.2 $\mathrm{kHz}$ in CW direction and $40 \mathrm{kHz}$ to $104.8 \mathrm{kHz}$ in CCW direction. The output power also monotonically increases from $0.95 \mathrm{~mW}$ to $2.88 \mathrm{~mW}$ in CW direction and $0.357 \mathrm{~mW}$ to $1.42 \mathrm{~mW}$ in CCW direction The phenomena that the repetition rate and output power of the Q-switched pulses increase with the elevation of the pump power are consistent with the typical characteristics of the Q-switched fiber laser [21]. To investigate the characteristics of the pulses in both directions, laser operating at $87.6 \mathrm{~mW}$ pump power is chosen for analysis. Fig. 3. (a) shows the optical spectra at $1560 \mathrm{~nm}$ single wavelength lasing scenario in both directions. The central wavelengths from CW and CCW directions are almost the 
same. However, spectrum in CCW direction is much smoother than the spectrum in CW direction. Moreover, irregular ripple oscillation can be found on the spectrum in CW direction. We speculate that this may be caused by the multimode oscillations and intracavity disturbances in CW direction [22]. The measured pulse width is $2.49 \mu$ s and $1.7 \mu \mathrm{s}$ in CW and CCW directions as depicted in Fig. 3. (b), respectively. The corresponding RF spectra of CW and CCW directions are shown in Fig. 3. (c) and Fig. 3. (d) individually manifesting that the Q-switched bidirectional fiber laser operates in a stable state. This is because the signal to noise ratio (SNR) is $41 \mathrm{~dB}$ at $66 \mathrm{kHz}$ in CW direction and $42.4 \mathrm{~dB}$ at 71.89 $\mathrm{kHz}$ in CCW direction [11]. Both insets of the Fig. 3. (c) and (d) are the RF spectra in $600 \mathrm{kHz}$ span under CW and CCW direction, respectively. This discrepancy in repetition rate, output power, pulse width and spectra is the result of laser cavity asymmetry which lead to different gain, loss, nonlinearity and output power in two directions [1].
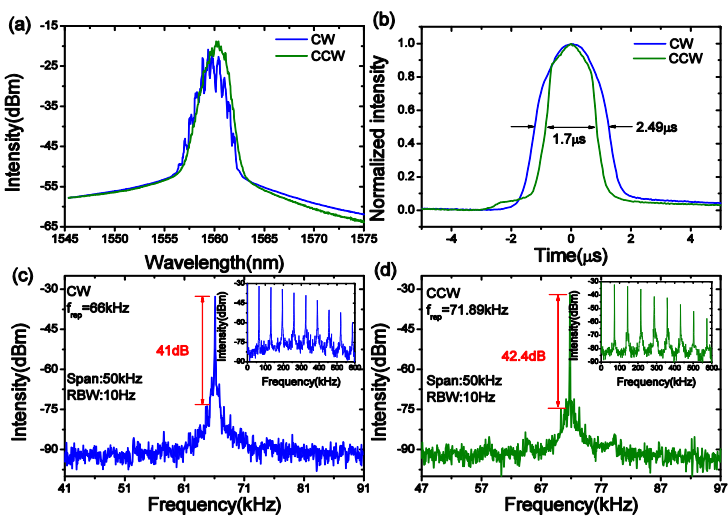

Fig. 3. (a) Output optical Spectra, (b) pulse width in CW and CCW direction; RF spectra (c) in CW direction and (d) in CW direction, inset: RF spectra in $600 \mathrm{kHz}$ span.

Under the same pump power of $87.6 \mathrm{~mW}$, we find that central wavelength in CW and CCW direction can be switched from 1560 $\mathrm{nm}$ to $1551 \mathrm{~nm}$ by adjusting two PCs as shown in Fig. 4. (a). The repetition rate and output power are $66.4 \mathrm{kHz}, 1.72 \mathrm{~mW}$ in CW direction, and $65.46 \mathrm{kHz}, 0.201 \mathrm{~mW}$ in CCW direction. Compared to lasing at $1560 \mathrm{~nm}$, the optical spectra at $1551 \mathrm{~nm}$ in both direction have narrower spectral contour which may be caused by lower nonlinearity, gain and larger cavity loss at $1551 \mathrm{~nm}$. The measured thresholds of Q-switched pulses generated in CW direction and CCW direction are $20 \mathrm{~mW}$ and $33.7 \mathrm{~mW}$, respectively, which are higher than those at $1560 \mathrm{~nm}$. Compared to the output power, both directions at $1551 \mathrm{~nm}$ are also lower than $1560 \mathrm{~nm}$ under pump power of $87.6 \mathrm{~mW}$. Through further adjusting PCs, dual-wavelength operation of $1560 \mathrm{~nm}$ and 1551 $\mathrm{nm}$ with a wavelength spacing of $9 \mathrm{~nm}$ are achieved in both directions as depicted in Fig. 4. (b). At this moment, the repetition rate and output power are $66.4 \mathrm{kHz}, 1.5 \mathrm{~mW}$ in CW direction, and $66.27 \mathrm{kHz}, 0.574 \mathrm{~mW}$ in CCW direction. Interestingly, as shown in Fig. 4. (c), dual-wavelength oscillation of $1560 \mathrm{~nm}$ and $1551 \mathrm{~nm}$ in CW direction, and single wavelength oscillation at $1560 \mathrm{~nm}$ in CCW direction can be simultaneously generated in the demonstrated bidirectional fiber laser. On the other hand, dual- wavelength oscillation of $1560 \mathrm{~nm}$ and $1551 \mathrm{~nm}$ in CCW direction, and single wavelength oscillation at $1551 \mathrm{~nm}$ in CW direction can also be simultaneously achieved in this bidirectional fiber laser as depicted in Fig. 4. (d). Additionally, these single/dual-wavelength switchable phenomena also can be achieved at other available pump powers by simply adjusting the PCs. The ability to generate single/dual-wavelength switching pulses in both CW and CCW directions makes this bidirectional fiber laser equivalent to two versatile laser sources.
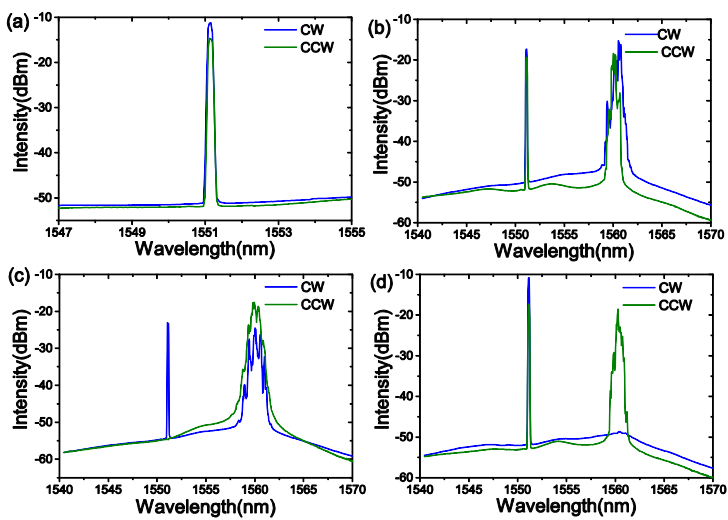

Fig. 4. Measured optical spectra of (a) Single wavelength (1551 $\mathrm{nm}$ ) in both directions, (b) dual-wavelength (1560 nm and $1551 \mathrm{~nm})$ in both direction, (c) dual-wavelength (1560 $\mathrm{nm}$ and $1551 \mathrm{~nm}$ ) in CW direction and single wavelength $(1560 \mathrm{~nm})$ in CCW direction, (d) dualwavelength (1560 nm and $1551 \mathrm{~nm})$ in CCW direction and single wavelength $(1551 \mathrm{~nm})$ in CW direction.

In our fiber laser, the cavity can be equivalent to a section of SMF with two bidirectional fiber polarizers ( $\left.45^{\circ} \mathrm{TFG}\right)$ at both ends. This structure can form a birefringent Lyot filter with a comb transmission spectrum[17]. Duo to the existence of the $45^{\circ} \mathrm{TFG}$, both in the CW and CCW direction of the fiber laser can obtained a similar birefringent Lyot filter. The typical transmission function of the filter can be described by the following equation [23]:

$\mathrm{T}=\cos ^{2} w_{1} \cos ^{2} w_{2}+\sin ^{2} w_{1} \sin ^{2} w_{2}$

$$
+\frac{1}{2} \sin \left(2 w_{1}\right) \sin \left(2 w_{2}\right) \cos \left(\Delta \emptyset_{L}\right) \text {. }
$$

where $w_{1}$ and $w_{2}$ are the angles between the polarization direction of the $45^{\circ} \mathrm{TFG}$ and the fast axis of the fiber. $\Delta \emptyset_{\mathrm{L}}=2 \pi \mathrm{L}\left(\mathrm{n}_{\mathrm{x}}\right.$ $\mathrm{n}_{\mathrm{y}}$ ) $/ \lambda$ is linear phase shift. The linear phase shift strongly depends on cavity length $\mathrm{L}$, strength of fiber birefringence $\Delta \mathrm{n}=\left(\mathrm{n}_{\mathrm{x}}-\mathrm{n}_{\mathrm{y}}\right)$. Here, we only consider linear phase shifts $\Delta \emptyset_{\mathrm{L}}$, because the nonlinear phase shift is much smaller compared to $\Delta \emptyset_{\mathrm{L}}$.

Fig. 5. (a) exhibits the simulated transmission spectra from $1520 \mathrm{~nm}$ to $1580 \mathrm{~nm}$ with different fiber birefringence $\Delta \mathrm{n}=3 \times 10^{-5}$, $5 \times 10^{-5}, 9 \times 10^{-5}$. It manifests that the smaller the fiber birefringence, the larger the wavelength spacing. When $\Delta \mathrm{n}$ is $5 \times 10^{-5}$, the wavelength spacing is $8.8 \mathrm{~nm}$ which is almost consistent with our dual-wavelength lasing experimental results with a wavelength spacing of $9 \mathrm{~nm}$. As shown in Fig. 5. (b), the calculated transmission spectra with different angles of $w_{1}$ and $w_{2}$ illustrate that peak position and modulation depth of the transmission spectra are related to angles of $w_{1}$ and $w_{2}$ which can be changed through rotating PCs. 

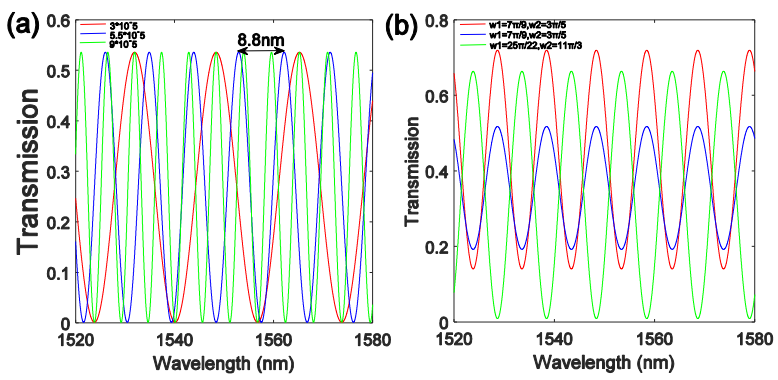

Fig. 5. The simulated transmission spectra with different (a) strength of fiber birefringence, and (b) angles of $\mathrm{w}_{1}$ and $\mathrm{w}_{2}$.

On the one hand, owing to the existence of comb transmission spectra of two fiber birefringent filters in $\mathrm{CW}$ and CCW directions, homogeneous gain broadening of the EDF at room temperature is suppressed and the gain profile is hence defined by the birefringent filter, which alleviates the mode competition and leads to the generation of dual-wavelengths. Furthermore, changing angles of $w_{1}$ and $w_{2}$ through rotating PCs to adjusting modulation depth, peak position of the transmission spectrum and fiber birefringence strength $\Delta \mathrm{n}$, the gain distribution and the wavelength obtaining maximum gain may also change. As a consequence, single wavelength of $1551 \mathrm{~nm}$ and $1560 \mathrm{~nm}$ can be emitted, and wavelength also can be switched between dualwavelength (1551 nm and $1560 \mathrm{~nm})$ and single wavelength (1551 $\mathrm{nm}$ or $1560 \mathrm{~nm}$ ).

On the other hand, due to the asymmetric structure of the bidirectional fiber laser, pulses pass through the cavity elements in reverse order in CW and CCW directions. As a result, gain, loss and nonlinearity are different in two directions, which combines with fiber birefringent filters in CW and CCW directions will result in different gain spectra in two directions. So, in both directions, the same wavelength will achieve different gain, which will cause the different wavelength lasing in $\mathrm{CW}$ and CCW directions. Hence, interesting phenomena that dual-wavelength $(1551 \mathrm{~nm}, 1560 \mathrm{~nm})$ in CW direction and single wavelength $(1560 \mathrm{~nm})$ in CCW direction as shown in Fig. 4 (c) or dual-wavelength $(1551 \mathrm{~nm}$, $1560 \mathrm{~nm})$ in CCW direction and single wavelength $(1560 \mathrm{~nm})$ in CW direction as shown in Fig. 4 (d) will be generated.

During the experiment, if we remove CNT/PVA composite film from laser cavity and let the $45^{\circ} \mathrm{TFG}$ based filter configuration remains in the bidirectional fiber laser. Regardless of changing power or adjusting PCs, the bidirectional fiber laser produces neither Q-switched pulses nor mode-locked pulses in both directions. When replacing the $45^{\circ} \mathrm{TFG}$ with SMF of the same length, the bidirectional fiber laser still operates in Q-switched state, but wavelength switching cannot be obtained and dualwavelength lasing also cannot be achieved. Consequently, in this bidirectional fiber laser, CNT/PVA composite film acts as SA to generate Q-switched pulses and $45^{\circ} \mathrm{TFG}$ based NPR configuration plays the role of inducing birefringence comb filter.

In conclusion, we have successfully demonstrated a single/dual-wavelength switchable bidirectional Q-switched fiber laser based on a bidirectional fiber polarizer ( $45^{\circ} \mathrm{TFG}$ ) and CNT-SA for the first time. Wavelength can be switched among single wavelength of $1551 \mathrm{~nm}, 1560 \mathrm{~nm}$ and dual-wavelength of 1560 $\mathrm{nm}$ and $1551 \mathrm{~nm}$ in both CW and CCW directions. Our scheme provides an effective way to achieve versatile dual-laser source and can reduce cost greatly in laser design. It also gives a guideline for achieving single/dual-wavelength switchable bidirectional Qswitched fiber laser in other wavelength. This fiber laser may find applications in sum-frequency mixing, synthesis of optical waveform, terahertz generation, optical communication and microwave radiation in the future.

Funding. National Natural Science Foundation of China (NSFC) (61605107,61505244); Young Eastern Scholar Program at Shanghai Institutions of Higher Learning (QD2015027); "Young 1000 Talent Plan" Program of China; The open program (Grant No.2017GZKF17) of the State Key Laboratory of Advanced Optical Communication Systems and Networks at Shanghai Jiaotong University, China; Open fund of Key Laboratory of Opto-electronic Information Technology, Ministry of Education ,Tianjin University, Tianjin 300072, P.R.China (2018KFKT009); RAEng/The Leverhulme Trust Senior Research Fellowships (LTSRF1617/13/57).

\section{References}

1. K. Kieu, M. Mansuripur, Opt. Lett. 33, 64-66 (2008).

2. S. Mehravar, R. A. Norwood, N. Peyghambarian, and K. Kieu, Appl. Phys. Lett. 108, 231104 (2016).

3. C. Ouyang, P. Shum, K. Wu, J. H. Wong, H. Q. Lam, and S. Aditya, Opt. Lett. 36, 2089-2091(2011).

4. C. Zeng, X.M Liu, and L. Yun, Opt. Express. 21, 18937-18942 (2013).

5. H. H. Liu, K. K. Chow, IEEE J. Sel. Top. Quantum Electron. 20, 278-282 (2014).

6. D. J. Li, D. Y. Shen, L. Li, H. Chen, D. Y. Tang, L. M. Zhao, Appl. Opt. 54, 7912-7916 (2015).

7. X. Zhao, Z. Zheng, Y. Liu, G. Q. Hu, J. S. Liu, Dual-Wavelength, IEEE Photonics Technol. Lett. 26, 1722-1725 (2014).

8. Y. F. Chen, S. W. Tsai, Opt. Lett. 27, 397-399 (2002).

9. N. D. Lai, F. Bretenaker, M. Brunel, J. Lightwave Technol. 21, 3037-3042 (2003).

10. L. Liu, Z. Zheng, X. Zhao, S. S. Sun, Y. S. Bian, Y. L. Su, J. S. Liu, J. S. Zhu, Opt. Commun. 294, 267-270 (2013).

11. J. M. Liu, Y. Chen, Y. Li, H. Zhang, S. Q. Zheng, and S. Xu,Photonics. Res. 6, 198-203 (2018).

12. Z. Y. Liu, Y. G. Liu, J. B. Du, S. Z. Yuan, and X. Y. Dong, Opt. Commun. 279, 168-172 (2007).

13. J. R. Qian, J. Su, L. Hong, Opt. Commun. 281, $4432-4434$ (2008).

14. M. P. FOK, C. Shu, Opt. Express. 15, 5925-5930 (2007).

15. Y. Z. Wang, J. F. Li, B. Zhai, Y. X. Hu, K. D. Mo, R. G. Lu, and Y. Liu, Opt. Express. 24, 15299-15306 (2016).

16. H. Zhang, D. Y. Tang, X. Wu, and L. M. Zhao, Opt. Express. 17,12692-12697 (2009).

17. Y. S. Fedotov, S. M. Kobtsev, R. N. Arif, A. G. Rozhin, C. B. Mou, and S. K. Turitsyn, Opt. Express. 20, 17797-17805 (2012).

18. S. P. Li , X. Chen, D. V. Kuksenkov, J. Koh, M. J Li, L. A. Zenteno, and D. A. Nolan, Opt. Express. 14, 6098-6102 (2006).

19. C. B. Mou, K. M. Zhou, L. Zhang, and I. Bennion, J. Opt. Soc. Am. B. 26, 1905-1911 (2009).

20. C. B. Mou, S. Sergeyev, A. Rozhin, and S. Turistyn, Opt. Lett. 36, 3831-3833 (2011).

21.Z. Q. Luo, M. Zhou, J. Weng, G. M. Huang, H. Y. Xu, C. C. Ye, and Z. P. Cai, Opt. Lett. 35, 3709-3911 (2010).

22. W. J. Cao, H. Y. Wang, A. P. Luo, Z. C. Luo, W. C. Xu, Laser Phys. Lett. 9, 54 58 (2012).

23. C. J. Chen, P. K. A. Wai, and C. R. Menyuk, Opt. Lett. 17, 417-419 (1992). 


\section{References}

1. K. Kieu, M. Mansuripur, All-fiber bidirectional passively mode-locked ring laser, Opt. Lett. 33, 64-66 (2008).

2. S. Mehravar, R. A. Norwood, N. Peyghambarian, and K. Kieu, Real-time dual-comb spectroscopy with a free-running bidirectionally mode-locked fiber laser, Appl. Phys. Lett. 108, 231104 (2016).

3. C. Ouyang, P. Shum, K. Wu, J. H. Wong, H. Q. Lam, and S. Aditya, Bidirectional passively mode-locked soliton fiber laser with a four-port circulator, Opt. Lett. 36, 2089-2091(2011).

4. C. Zeng, X.M Liu, and L. Yun, Bidirectional fiber soliton laser mode-locked by single-wall carbon nanotubes, Opt. Express. 21, 18937-18942 (2013).

5. H. H. Liu, K. K. Chow, Operation-switchable bidirectional pulsed fiber laser incorporating carbon-nanotube-based saturable absorber, IEEE J. Sel. Top. Quantum Electron. 20, 278-282 (2014).

6. D. J. Li, D. Y. Shen, L. Li, H. Chen, D. Y. Tang, L. M. Zhao, Unidirectional dissipative soliton operation in an all-normal-dispersion Yb-doped fiber laser without an isolator, Appl. Opt. 54, 7912-7916 (2015).

7. X. Zhao, Z. Zheng, Y. Liu, G. Q. Hu, J. S. Liu, Dual-Wavelength, Bidirectional Single-Wall Carbon Nanotube Mode-Locked Fiber Laser, IEEE Photonics Technol. Lett. 26, 1722-1725 (2014).

8. Y. F. Chen, S. W. Tsai, Diode-pumped Q-switched $\mathrm{Nd} \mathrm{YVO}_{4}$ yellow laser with intracavity sum-frequency mixing, Opt. Lett. 27, 397-399 (2002).

9. N. D. Lai, F. Bretenaker, M. Brunel, Coherence of pulsed microwave signals carried by two-frequency solid-state lasers, J. Lightwave Technol. 21, 3037-3042 (2003).

10. L. Liu, Z. Zheng, X. Zhao, S. S. Sun, Y. S. Bian, Y. L. Su, J. S. Liu, J. S. Zhu, Dualwavelength passively Q-switched Erbium doped fiber laser based on an SWNT saturable absorber, Opt. Commun. 294, 267-270 (2013).

11. J. M. Liu, Y. Chen, Y. Li, H. Zhang, S. Q. Zheng, and S. Xu, Switchable dualwavelength Q-switched fiber laser using multilayer black phosphorus as a saturable absorber, Photonics. Res. 6, 198-203 (2018).

12.Z. Y. Liu, Y. G. Liu, J. B. Du, S. Z. Yuan, and X. Y. Dong, Switchable triplewavelength erbium-doped fiber laser using a single fiber Bragg grating in polarization-maintaining fiber, Opt. Commun. 279, 168-172 (2007).

13. J. R. Qian, J. Su, L. Hong, A widely tunable dual-wavelength erbium-doped fiber ring laser operating in single longitudinal mode, Opt. Commun. 281, 4432-4434 (2008).

14. M. P. FOK, C. Shu, Tunable dual-wavelength erbium-doped fiber laser stabilized by four-wave mixing in a $35-\mathrm{cm}$ highly nonlinear bismuth-oxide fiber, Opt. Express. 15, 5925-5930 (2007).

15. Y. Z. Wang, J. F. Li, B. Zhai, Y. X. Hu, K. D. Mo, R. G. Lu, and Y. Liu, Tunable and switchable dual-wavelength mode-locked $\mathrm{Tm}^{3+}$-doped fiber laser based on a fiber taper, Opt. Express. 24, 15299-15306 (2016).

16. H. Zhang, D. Y. Tang, X. Wu, and L. M. Zhao, Multi-wavelength dissipative soliton operation of an erbium-doped fiber laser, Opt. Express. 17,1269212697 (2009).

17. Y. S. Fedotov, S. M. Kobtsev, R. N. Arif, A. G. Rozhin, C. B. Mou, and S. K. Turitsyn, Spectrum-, pulsewidth-, and wavelength-switchable all-fiber mode-locked Yb laser with fiber based birefringent filter, Opt. Express. 20, 17797-17805 (2012).

18. S. P. Li , X. Chen, D. V. Kuksenkov, J. Koh, M. J Li, L. A. Zenteno, and D. A. Nolan, Wavelength tunable stretched-pulse mode-locked all-fiber erbium ring laser with single polarization fiber, Opt. Express. 14, 6098-6102 (2006).

19. C. B. Mou, K. M. Zhou, L. Zhang, and I. Bennion, Characterization of $45^{\circ}-$ tilted fiber grating and its polarization function in fiber ring laser, J. Opt. Soc. Am. B. 26, 1905-1911 (2009).

20. C. B. Mou, S. Sergeyev, A. Rozhin, and S. Turistyn, All-fiber polarization locked vector soliton laser using carbon nanotubes, Opt. Lett. 36, 38313833 (2011).

21.Z. Q. Luo, M. Zhou, J. Weng, G. M. Huang, H. Y. Xu, C. C. Ye, and Z. P. Cai, Graphene-based passively Q-switched dualwavelength erbium-doped fiber laser, Opt. Lett. 35, 3709-3911 (2010).
22. W. J. Cao, H. Y. Wang, A. P. Luo, Z. C. Luo, W. C. Xu, Graphene-based, 50 $\mathrm{nm}$ wide-band tunable passively Q-switched fiber laser, Laser Phys. Lett. 9, 54-58 (2012).

23. C. J. Chen, P. K. A. Wai, and C. R. Menyuk, Soliton fiber ring laser, Opt. Lett. 17, 417-419 (1992). 